\title{
Total nephroureterocystectomy and urethrectomy due to urothelial carcinoma associated with the BK polyomavirus infection after kidney transplantation: a case report with literature review
}

Ryoichi Maenosono ${ }^{1,3}$, Masayoshi Okumi $^{1}$, Kohei Unagami ${ }^{2,4^{*}}$ (D), Hironori Fukuda', Kazuhiko Yoshida', Yoichi Kakuta', Toshio Takagi', Junpei lizuka', Tomokazu Shimizu', Haruhito Azuma ${ }^{3}$, Yoji Nagashima5 Kazunari Tanabe ${ }^{1}$, Kosaku Nitta ${ }^{2}$ and Hideki Ishida ${ }^{4}$

\begin{abstract}
Background: BK polyomavirus (BKPyV) infection after kidney transplantation is an important cause of graft failure among kidney transplant recipient and may cause malignant tumor, although the association between BKPyV infection and malignant tumor has been controversial yet.
\end{abstract}

Case presentation: We report a case of a 39-year-old-male kidney transplantation (KTx) recipient with urine BKPyV replication who developed a graft pelvic tumor with the positive Simian virus 40 large T antigen (SV40 TAg). The patients received a living-related KTx from his 65-year-old mother. A protocol biopsy at 14 months after KTx showed BKPyV-associated nephropathy. Therefore, the dose of immunosuppressants was reduced, resulting in improved BKPyV viremia, but viruria persisted. About 117 months after KTx, urine cytology showed atypical cells suspicious for malignancy. Cystoscopy revealed a tumor on the neck of the bladder. Transurethral resection of the bladder tumor (TUR-BT) was performed; however, the diagnosis of malignancy was not confirmed at that time. Six months after the TUR-BT, urine cytology showed atypical cells definite for malignancy. Computed tomography and retrograde pyelography showed no evidence of urinary tract tumor and metastasis. Subsequently, total

nephroureterocystectomy and urethrectomy were performed. Histological examination of the graft ureter revealed a high-grade urothelial carcinoma, with glandular differentiation, pT1. Immunohistochemically, the tumor showed positivities for SV40 TAg and p53, along with increased Ki67 labeling cells were increased. By contrast, nonneoplastic cells were negative for SV40 TAg. At the time of writing the present manuscript, the patient is free from recurrence or residual tumor and being closely monitored without additional therapy, 32 months after the surgery.

(Continued on next page)

\footnotetext{
* Correspondence: unagami.kohei@twmu.ac.jp

${ }^{2}$ Department of Urology, Osaka Medical College, Osaka, Japan

${ }^{4}$ Departments of Organ Transplant, Tokyo Women's Medical University, 8-1

Kawada-cho, Shinjuku-ku, Tokyo 162-8666, Japan

Full list of author information is available at the end of the article
}

\section{$\triangle B M C$}

(c) The Author(s). 2020 Open Access This article is licensed under a Creative Commons Attribution 4.0 International License, which permits use, sharing, adaptation, distribution and reproduction in any medium or format, as long as you give appropriate credit to the original author(s) and the source, provide a link to the Creative Commons licence, and indicate if changes were made. The images or other third party material in this article are included in the article's Creative Commons licence, unless indicated otherwise in a credit line to the material. If material is not included in the article's Creative Commons licence and your intended use is not permitted by statutory regulation or exceeds the permitted use, you will need to obtain permission directly from the copyright holder. To view a copy of this licence, visit http://creativecommons.org/licenses/by/4.0/ The Creative Commons Public Domain Dedication waiver (http://creativecommons.org/publicdomain/zero/1.0/) applies to the data made available in this article, unless otherwise stated in a credit line to the data. 
(Continued from previous page)

Conclusion: The relationship between BKPyV infection after KTx and bladder carcinogenesis remains to be elucidated. However, when the KTx recipients who continue to have BKPyV infection for a long time are treated, the possibility of risk factors for renourinary carcinoma should always be carefully considered.

Keywords: BK polyomavirus, Urothelial carcinoma, Kidney transplantation, Total nephroureterocystectomy, Urethrectomy

\section{Background}

In recent years, long-term graft survival for kidney transplantation (KTx) has improved with advances in immunosuppressive therapy. Currently, the widely used strategy for immunosuppression related with $\mathrm{KTx}$ is suppression of helper $\mathrm{T}$ lymphocyte activity such as tacrolimus (TAC)-based immunosuppression; viral infection is considered as a frequent complication after KTx. In particular, $\mathrm{BK}$ polyomavirus (BKPyV) is an important cause of nephropathy and graft failure for KTx recipients. BKPyV infection may cause nephropathy in $2-10 \%$ of KTx recipients [1-5] and lead to graft loss in approximately 15-80\% [1, 5-9]. Furthermore, BKPyV has been known to have a tropism for the genitourinary tract and causes clinical disease. Thus, for immunocompromised patients such as KTx recipients, not only BKPyVassociated nephropathy but also ureteral stenosis and hemorrhagic cystitis would occur.

Recently, the relationship between BKPyV and genitourinary malignant tumor, such as urothelial carcinoma $[10,11]$ especially in bladder cancer [11-13] and renal cell carcinoma [14], has been reported. However, few studies suggested a potential role of BKPyV in the development of urologic tract malignancies in KTx recipients [15], and the association remains controversial. Herein, we describe a case of a KTx recipient with positive urine $\mathrm{BKPyV}$ replication who developed a graft pelvic tumor with positive immunohistochemical staining for simian virus 40 large $\mathrm{T}$ antigen (SV40 TAg).

\section{Case presentation}

A 38-year-old man was diagnosed with chronic kidney disease and underwent hemodialysis. The etiology of his renal failure was unknown. His past medical history included hypertension alone, without a history of malignancy or any exposure to carcinogens. At 39 years of age, the patient received $\mathrm{ABO}$-incompatible living-donor KTx. The donor was his 65-year-old mother. Human leukocyte antigen typing revealed two mismatches, and donor-specific antibody tests were negative. Immunosuppressive therapy comprised TAC, mycophenolate mofetil (MMF), methylprednisolone (MP), basiliximab, and rituximab. Plasmapheresis was also performed thrice because of ABO incompatibility. Serum creatinine (sCre) level decreased to $1.76 \mathrm{mg} / \mathrm{dL}$ on postoperative day
(POD) 5. A protocol biopsy on POD 17 showed no evidence of rejection, and he was discharged on POD 23. Thereafter, TAC, MMF, and MP were continued as immunosuppressive maintenance therapy. Furthermore, his sCre level ranged from 2.0 to $2.4 \mathrm{mg} / \mathrm{dL}$. At 14 months after $\mathrm{KTx}$, his graft function became stable (sCre, 2.49); however, the protocol biopsy showed BKPyV-associated nephropathy (BKPyVAN) (Fig. 1) incidentally. In addition, polymerase chain reaction (PCR) with urine sample detected BKPyV at $4.9 \times 10^{7}$ copies/mL; BKPyV was undetectable in serum PCR test. Therefore, we decreased the dosage of TAC and switched MMF to mizoribine (MZ). Thereafter, the PCR titer of urine BKPyV decreased but persisted (Fig. 2). 98 months after KTx, hematuria was suddenly observed and persisted (urine red blood cell level varied between 10-19 and 50-99/high-power field). Thus, urine cytology was repeated during the follow-up: the patient was noted to suspicious of malignancy by urine cytology and cystoscopy revealed tumor on the neck of the bladder at 117 months after KTx (Fig. 3). Transurethral resection of the bladder tumor (TUR-BT) was performed; however, we failed to find the tumor resection and thereby definitive diagnosis was not reached. Thereafter, urine cytological examination was periodically continued, and 6 months after TUR-BT, bladder, and graft urine cytology showed malignant cells (Fig. 4). Although computed tomography and retrograde pyelography showed no significant evidence of urinary tract tumor and metastasis, the patient was finally diagnosed as malignancy. Thereafter, total nephroureterocystectomy and urethrectomy were performed to radically resect the malignant tumor at 124 months after KTx. Grossly, there was a papillary tumor in the uretero-pelvic junction on the kidney graft (Fig. 5). Histologically, the tumor was diagnosed as a high-grade urothelial carcinoma with glandular differentiation, pT1, in the ureter of the allograft. Immunohistochemically, SV40 TAg and p53 were positive in the tumor cells. Ki67- was increased. Nonneoplastic cells failed to show a positive reaction for SV40 TAg staining (Fig. 6). The patient resumed maintenance hemodialysis after the operation. To date, the patient is closely monitored without additional therapy and without recurrence or metastasis for 32 months. 


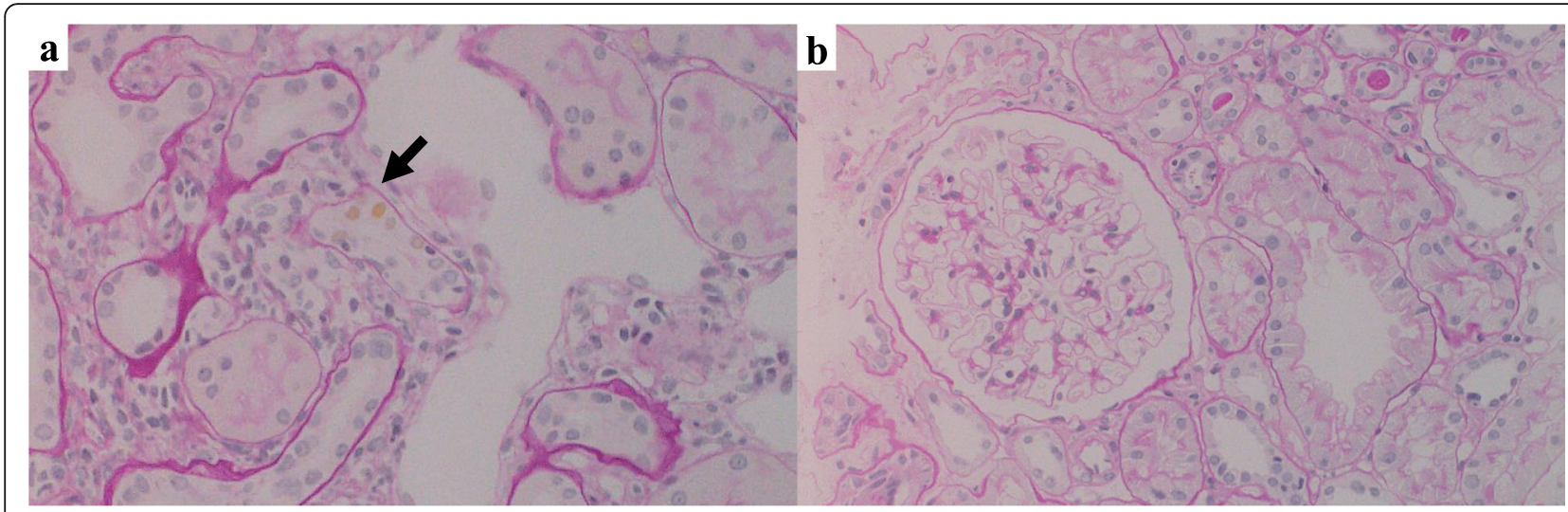

Fig. 1 Protocol biopsy of transplanted kidney performed on postoperative day 426. a BK polyomavirus-associated nephropathy (arrow) and $\mathbf{b}$ no result of rejection findings [a Simian vacuolating virus 40 large $T$ antigen stain, and $\mathbf{b}$ hematoxylin-eosin stain]

\section{Discussion and conclusions}

BKPyV primary infection commonly occurred as an asymptomatic, upper respiratory tract infection, or flulike infection at childhood, and approximately $65-90 \%$ of the general population may reach a seroprevalence state $[5,16-20]$; thereafter, the virus is considered to remain mainly in the urinary tract. BKPyV infection in KTx recipient develops not only renal dysfunction due to nephropathy but also ureteral stenosis and hemorrhagic cystitis [21]. Moreover, BKPyV has been recently reported as a viral tumor that occurs in immunocompromised hosts such as KTx recipients, together with a long-term allograft survival. The exact effect of $\mathrm{BKPyV}$ in KTx recipients remains uncertain; however, the incidence of urinary tract tumor such as bladder tumor and renal cell carcinoma would increase [10-14] throughout BKPyV replication [22, 23].

In general, BKPyVAN showed a positive immunohistochemical staining for SV40 TAg, p53, and Ki67 throughout the upregulation of these markers [24]. However, the role of $\mathrm{BKPyV}$ in solid organ recipients is uncertain at present, but urinary tract tumors are among the best candidates for an etiologic association with BKPyV infection. In vitro studies, BKPyV infection could transform

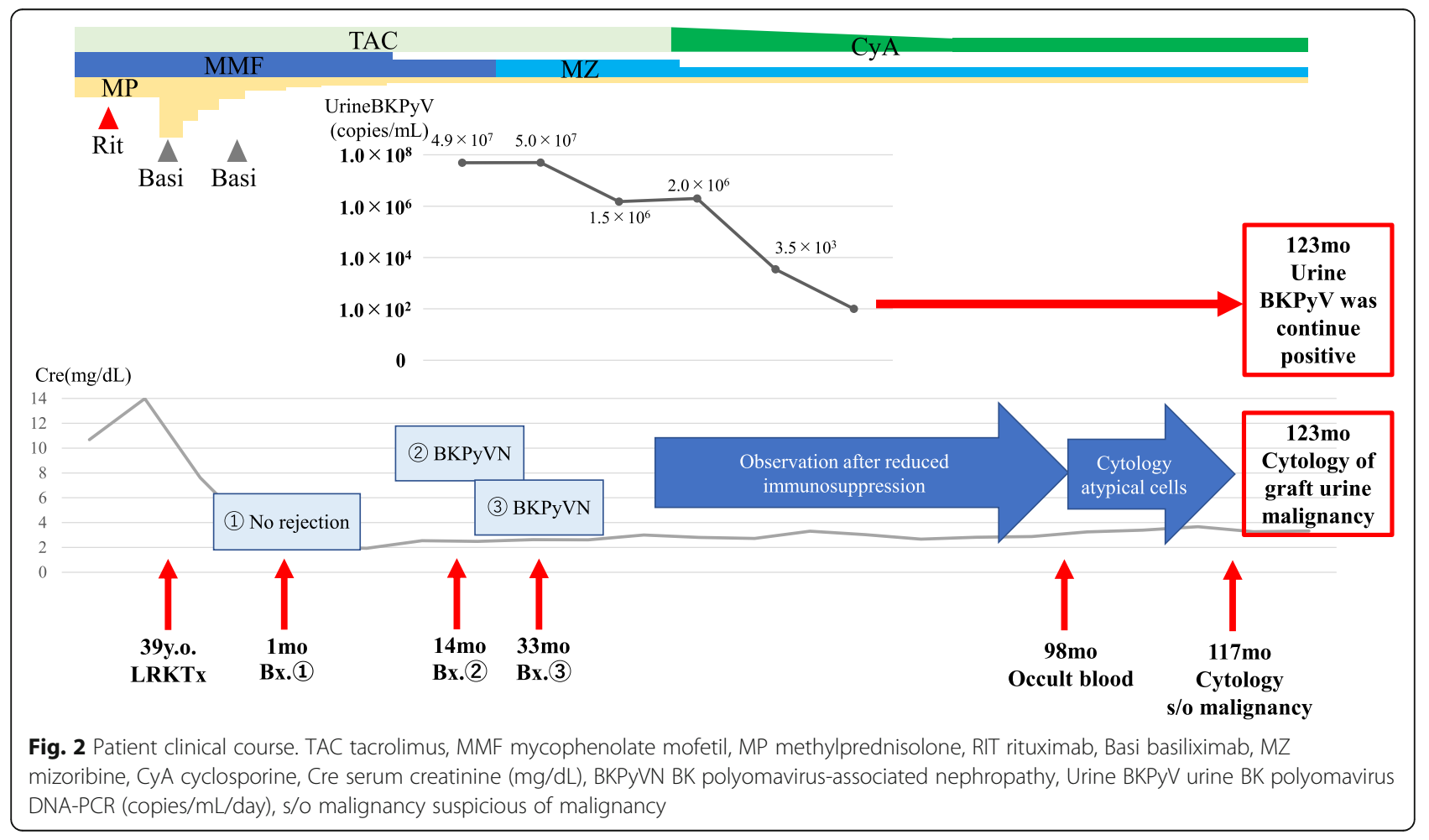




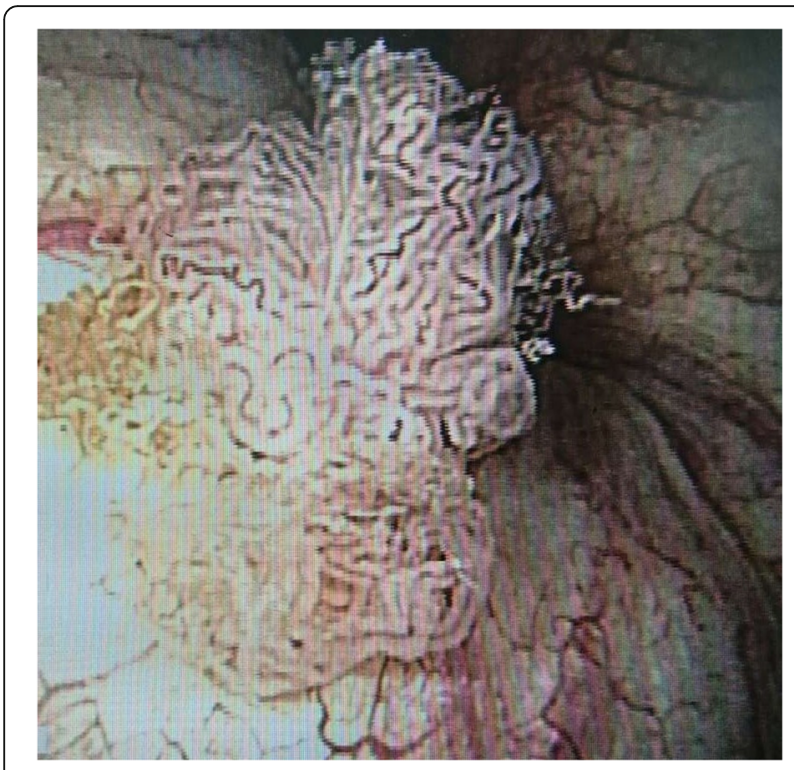

Fig. 3 Cystoscopy 117 months after kidney transplantation. Cystoscopy showed the papillary tumor existed in the bladder neck

cells and cause malignancy in immunosuppressed or immature rodents. BKPyV genomes are divided into three distinct regions: the early viral gene region (EVGR), the late viral gene region (LVGR), and the noncoding control region. EVGR encodes the regulatory proteins large tumor antigen (LTAg), which inactivates the tumor suppressors, pRB and p53 [23]. Kenan et al. also hypothesized that dysregulation of LTag by gene integration of $\mathrm{BKPyV}$ with persistent overexpression promotes cell growth, genetic instability, neoplastic transformation,

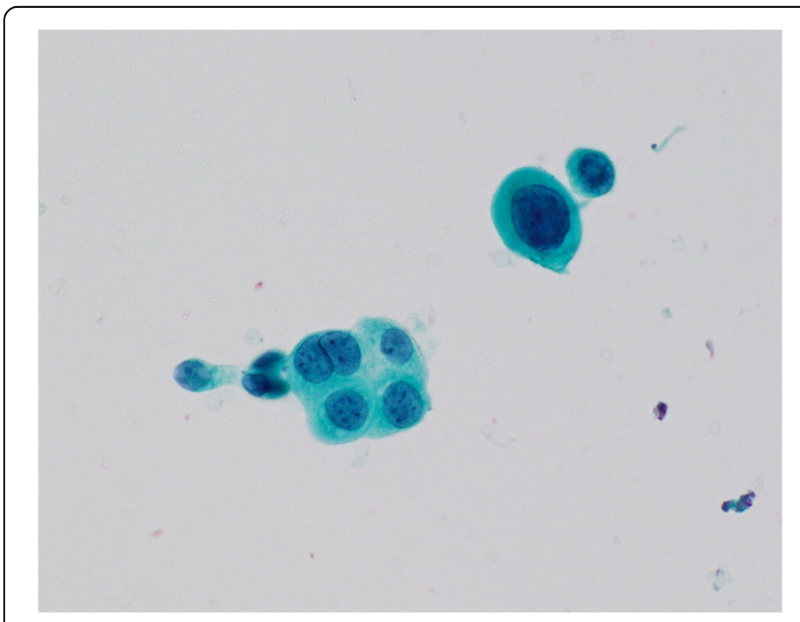

Fig. 4 Cytology 123 months after kidney transplantation. The bladder and graft urine cytology showed malignant cells, class IV. Hyperchromatic atypical cells with anisocytosis and nuclear enlargement were found, without virus-infected cells (decoy cells). [cytology from the bladder urine; papanicolaou stain] and tumorigenesis [25]. These oncogeneses occur mainly in renourinary organs, including the ureter [23], as in our case. Furthermore, Monini et al. reported that the presence and physical state of BKPyV DNA LTAg gene sequences were amplified by PCR from the prostate, kidney, ureter, and bladder, including these carcinomas [26]. Taking this into consideration, SV40 TAg positivity in tissues indicated $\mathrm{BKPyV}$ infection status, and past $\mathrm{BKPyV}$ infection may influence malignancy development; thus, SV40 TAg positivity in tissues is associated with BKPyV infectious carcinoma, although not always.

In $\mathrm{KTx}$ recipients receiving strong immunosuppressive therapy, viral infection would be a serious problem. Thus, once BKPyV infection is found detected, the reduction of immunosuppressive drugs is recommended [17]. Especially, TAC is a current key drug mainly used as an immunosuppressant for $\mathrm{KTx}$ recipients [27]. TAC is identified as a risk factor for developing $\mathrm{BKPyV}$ viremia or BKPyVAN [3, 28-30], which may reduce the TAC dosage [31]. In this report, the recipient was managed with maintenance immunosuppression consisting of TAC, MMF, and MP, but a protocol biopsy performed at 14 months after $\mathrm{KTx}$ revealed subclinical $\mathrm{BKPyV}$-associated nephropathy; thereafter, TAC reduction and anti-metabolite medicine conversion were performed as immunosuppression weakening. After these changes, the PCR titer in urine BKPyV was decreased but remained.

In this case, malignancy was detected about 10 years after KTx. Although during this long duration, the graft kidney might have been exposed to exogenous carcinogens, e.g., smoking or chemical dyes such as aromatic amines, ionizing radiation, and cyclophosphamide, only $\mathrm{BKPyV}$ viruria was suspected as the risk factor for malignancy. Urine cytology was routinely performed, and the cellular atypism got higher and finally diagnosed as bladder cancer based on cystoscopy findings. At first, TURBT on the neck of the bladder was performed; however, we failed to catch the tumor resection and characteristic evaluation was not performed. Thereafter, repeat urine cytology was performed after TUR-BT to detect malignancy. The grade of malignancy based on urine cytology worsened and finally diagnosing allograft-ureter cancer. Although intravesical Bacillus Calmette-Guerin instillation was considered at that time, the treatment had been abandoned because of the patient status under immunosuppressant. In order to improve the curability, total nephroureterocystectomy and urethrectomy were performed at 124 months after KTx in spite of stable graft function.

For immunochemical staining, urothelial carcinoma was stained positive with SV40 TAg; however, normal tissues, such as native kidney and ureter, and allograft kidney tissues were negative with SV40 TAg staining. 

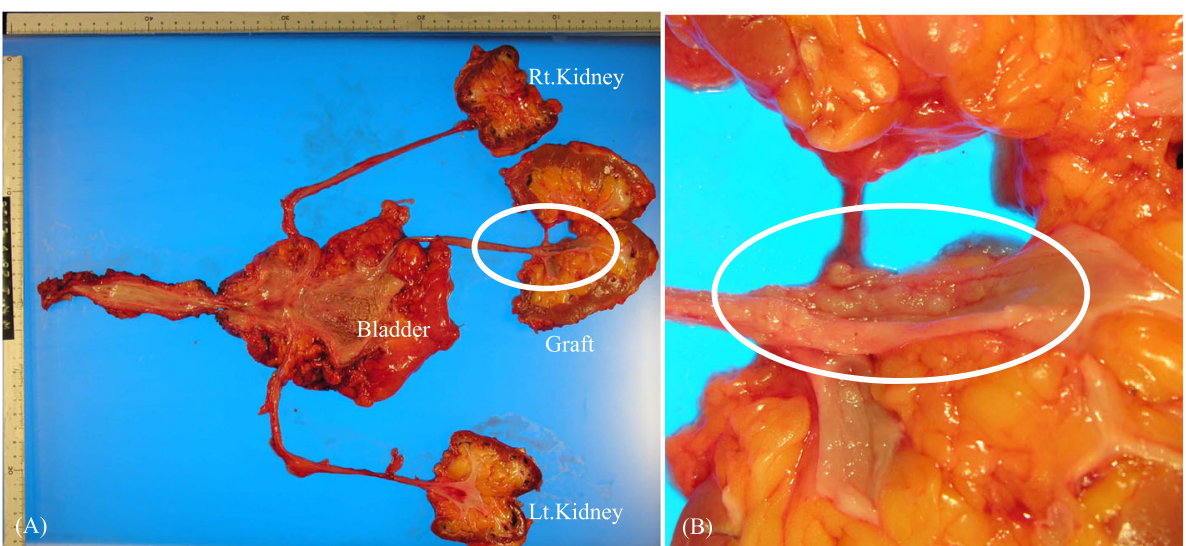

Fig. 5 Total nephroureterocystectomy and urethrectomy. a Urinary tract without graft did not show the tumor, and $\mathbf{b}$ approximately 10-mmdiameter tumor was located at the graft pelvis

SV40 TAg staining is a reliable test for BKPyV infection [32]; therefore, these results suggested that BKPyV infection for a long-term made graft urothelium malignant, and the focus of malignancy was allograft ureter clearly.

As regards the study limitation, detail DNA analysis or fluorescence in situ hybridization was not performed; however, SV40 TAg-positive cells in this case were detected only in the carcinoma tissue, and other tissues were not stained with immunohistochemical staining. Furthermore, we unfortunately failed to catch the bladder tumor during the TUR-BT. Therefore, the relationship between bladder tumor and $\mathrm{BKPyV}$ infection remains unclear.

In conclusion, an interesting but challenging case of urothelial carcinoma in allograft is presented for KTx recipients after BKPyV-associated nephropathy with remaining viruria. When the KTx recipients who continue to have BKPyV infection for a long time are treated, the possibility of risk factors not only for BKPyV-associated nephropathy but also for renourinary carcinoma should always be carefully considered. Therefore, we continued careful follow-up by routine urine

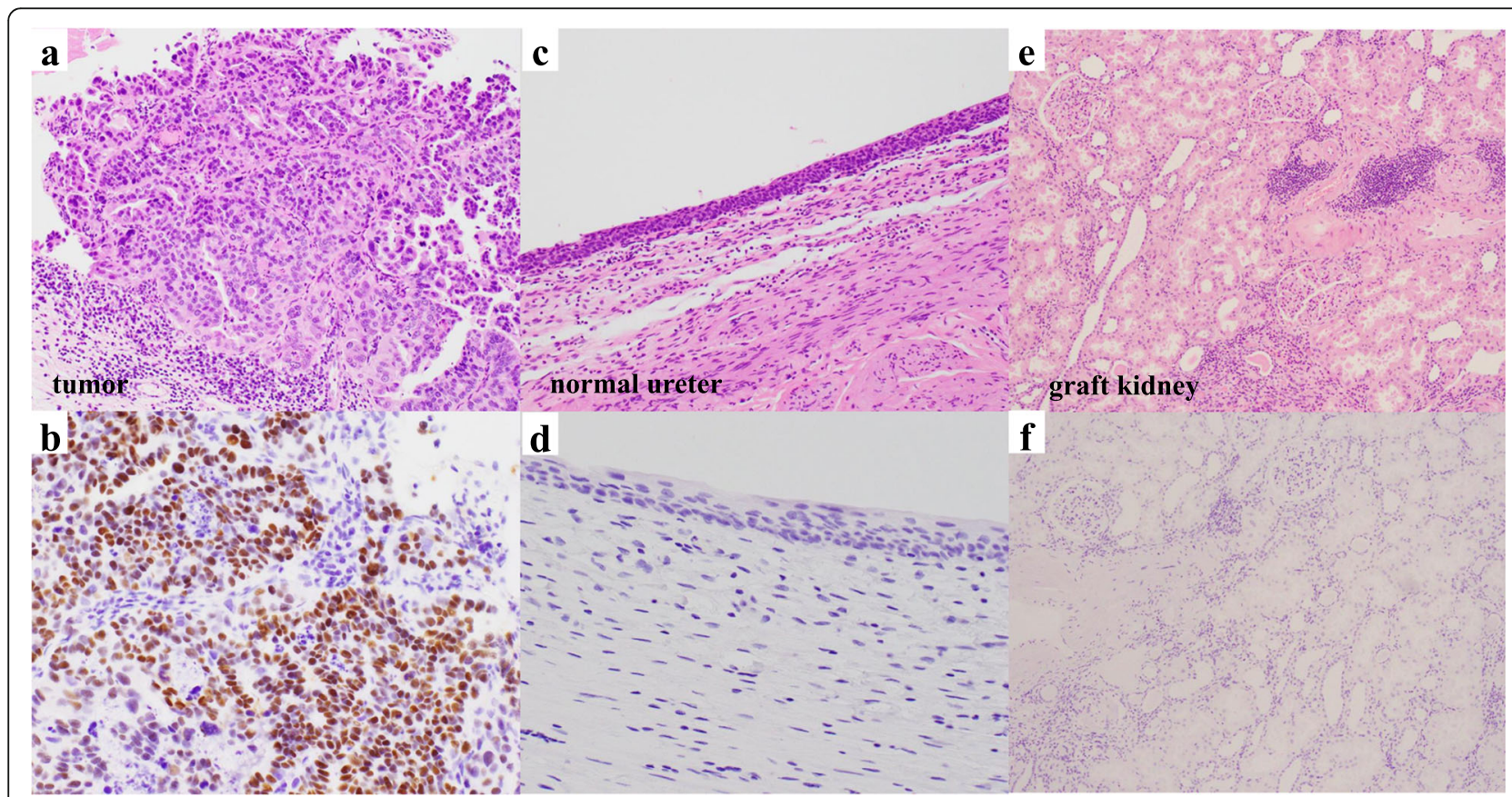

Fig. $\mathbf{6}$ Light microscopy findings from the allograft and native kidney. [a, $\mathbf{b}$ tumor from the allograft ureter; $\mathbf{c}, \mathbf{d}$ normal tissue from the native kidney ureter; (e, f normal tissue from graft kidney; a, c, e hematoxylin-eosin stain; b, d, f Simian vacuolating virus 40 large T antigen (SV40 TAg) stain]. b Tumor from the allograft ureter showed positive for SV40 TAg staining. $\mathbf{d}$ Other normal tissues were not positive for SV40 TAg staining 
cytology tests, and the patient was immediately diagnosed with urinary tract carcinoma and total nephroureterocystectomy and urethrectomy were performed.

\section{Literature review}

As treatment strategies for $\mathrm{BKPyV}$ infection, reducing or stopping immunosuppressive agents, administering intravenous immunoglobulin, cidofovir induction, and others may be considered.

Their treatment efficacies are unclear, and acute rejection may occur from reduced immunosuppressive therapy. There are no antiviral drugs; therefore, screening for BKPyV replication and timely reduction of immunosuppression as the preemptive intervention is currently recommended [31, 33, 34]. Significant BKPyV replication manifests as (a) probable BKPyVAN, defined by sustained BKPyV viremia of $>3 \log _{10}$ copies $/ \mathrm{mL}$ in two measurements within 3 weeks; or (b) presumptive BKPyVAN, defined by at least one of two measurements are > $4 \log _{10}$ copies/mL; or (c) proven BKPyVAN, according to biopsy results [34].

For reduction immunosuppression, two strategies have been recommended by the American Society of Transplantation guidelines: (i) dose reduction of calcineurin inhibitor (CNI) by $25-50 \%$ in one or two steps, followed by reducing antimetabolites by $50 \%$, followed by discontinuing the latter and (ii) reduction of antimetabolites by $50 \%$, followed by reducing CNI by $25-50 \%$, and then discontinuing antimetabolites. Bischof et al. reported that the "CNI first" immunosuppression reduction strategy leads to similar long-term outcomes in patients with and without BKPyV viremia and is associated with a low risk for clinical antibody-mediated rejection after clearance of BKPyV viremia [31]. In addition, prednisone is typically tapered to $10 \mathrm{mg}$ or less per day [34].

In the standard therapy, the conversion from MMF to $\mathrm{MZ}$ is not included because MZ is not available worldwide. In Japan, immunosuppression therapy, including $\mathrm{MZ}$, is used for a compromised host for viral infections such as cytomegalovirus $[35,36]$. Therefore, MZ is sometimes administered instead of MMF for the inhibition of BKPyV infection [37, 38].

$\mathrm{T}$ cell- and antibody-mediated rejection can occur complicated with BKPyV infection in tandem with immunosuppression reduction. It is required to judge which one is mainly a factor of deterioration of allograft function and may change the treatment strategy, such as whether immunosuppression should be re-increased or not.

\section{Abbreviations}

KTx: Kidney transplantation; TAC: Tacrolimus; BKPyV: BK polyomavirus; SV40 TAg: Simian virus 40 large T antigen; MMF: Mycophenolate mofetil; MP: Methylprednisolone; sCre: Serum creatinine; POD: Postoperative day; PCR: Polymerase chain reaction; BKPyVAN: PyV-associated nephropathy; TURBT: Transurethral resection of the bladder tumor; CNI: Calcineurin inhibitor

\section{Acknowledgements}

Not applicable.

Authors' contributions

R.M., M.O., K.U., Y.N., and H.I. participated in research design, writing the article, and performance of the research. H.F., K.Y., Y.K., T.T., J.I., T.S., H.A., K.T., and K.N. contributed to the design and editing of the article. The authors read and approved the final manuscript.

\section{Funding}

The authors confirm that they received no funding for this study.

Availability of data and materials

All data analyzed during this study are included in this published article.

Ethics approval and consent to participate

The authors have no ethical conflicts to disclose. The present case report was approved by our institution's Institutional Review Board.

Consent for publication

Written informed consent was obtained from the patient.

\section{Competing interests}

The authors have no competing interests and do not have any relationships to disclose.

\section{Author details}

${ }^{1}$ Urology, Kidney Center, Tokyo Women's Medical University, Tokyo, Japan. ${ }^{2}$ Department of Urology, Osaka Medical College, Osaka, Japan. ${ }^{3}$ Nephrology, Kidney Center, Tokyo Women's Medical University, Tokyo, Japan.

${ }^{4}$ Departments of Organ Transplant, Tokyo Women's Medical University, 8-1 Kawada-cho, Shinjuku-ku, Tokyo 162-8666, Japan. ${ }^{5}$ Department of Surgical Pathology, Tokyo Woman's Medical University, Tokyo, Japan.

Received: 7 May 2020 Accepted: 28 October 2020

Published online: 13 November 2020

\section{References}

1. Masutani K. Viral infections directly involved in kidney allograft function. Nephrology. 2018;23(Suppl 2):31-7.

2. Masutani K, Shapiro R, Basu A, et al. Putative episodes of T-cell-mediated rejection in patients with sustained BK viruria but no viremia. Transplantation. 2012;94:43-9.

3. Gard L, van Doesum W, Niesters HGM, et al. A delicate balance between rejection and BK polyomavirus associated nephropathy: a retrospective cohort study in renal transplant recipients. PLoS One. 2017;12:e0178801.

4. Reischig $T$, Kacer M, Hes $O$, et al. Viral load and duration of BK polyomavirus viraemia determine renal graft fibrosis progression: histologic evaluation of late protocol biopsies. Nephrol Dial Transplant. 2019;34:1970-8.

5. Kotla SK, Kadambi PV, Hendricks AR, et al. BK polyomavirus-pathogen, paradigm and puzzle. Nephrol Dial Transplant. 2019; Online ahead of print.

6. Wadei HM, Rule AD, Lewin M, et al. Kidney transplant function and histological clearance of virus following diagnosis of polyomavirusassociated nephropathy (PVAN). Am J Transplant. 2006;6:1025-32.

7. Drachenberg $C B$, Beskow $C O$, Cangro $C B$, et al. Human polyoma virus in renal allograft biopsies: morphological findings and correlation with urine cytology. Hum Pathol. 1999;30:970-7.

8. Howell DN, Smith SR, Butterly DW, et al. Diagnosis and management of BK polyomavirus interstitial nephritis in renal transplant recipients. Transplantation. 1999:68:1279-88.

9. Ramos E, Drachenberg CB, Papadimitriou JC, et al. Clinical course of polyoma virus nephropathy in 67 renal transplant patients. J Am Soc Nephrol. 2002;13:2145-51.

10. Salvatore SP, Myers-Gurevitch PM, Chu S, et al. Polyoma (BK) virus associated urothelial carcinoma originating within a renal allograft five years following resolution of polyoma virus nephropathy. Clin Nephrol. 2016;85:179-83. 
11. Gupta G, Kuppachi S, Kalil RS, et al. Treatment for presumed BK polyomavirus nephropathy and risk of urinary tract cancers among kidney transplant recipients in the United States. Am J Transplant. 2018; 18:245-52.

12. Grippo PJ, Sandgren EP. Highly invasive transitional cell carcinoma of the bladder in a simian virus 40 T-antigen transgenic mouse model. Am J Pathol. 2000;157:805-13

13. Zhang ZT, Pak J, Shapiro E, et al. Urothelium-specific expression of an oncogene in transgenic mice induced the formation of carcinoma in situ and invasive transitional cell carcinoma. Cancer Res. 1999:59:3512-7.

14. Neirynck V, Claes K, Naesens M, et al. Renal cell carcinoma in the allograft: what is the role of polyomavirus? Case Rep Nephrol Urol. 2012:2:125-34.

15. Meier RPH, Müller YD, Dietrich PY, et al. Immunologic clearance of a BK virus-associated metastatic renal allograft carcinoma. Transplantation. 2020; [Epub ahead of print].

16. Knowles WA. Discovery and epidemiology of the human polyomaviruses BK virus (BKV) and JC virus (JCV). Adv Exp Med Biol. 2006;577:19-45.

17. Hirsch HH, Steiger J. Polyomavirus BK. Lancet Infect Dis. 2003;3(10):611-23.

18. Stolt A, Sasnauskas K, Koskela P, et al. Seroepidemiology of the human polyomaviruses. J Gen Virol. 2003;84:1499-504.

19. Flaegstad T, Ronne K, Filipe AR, et al. Prevalence of anti BK virus antibody in Portugal and Norway. Scand J Infect Dis. 1989;21:145-57.

20. Hariharan S. BK virus nephritis after renal transplantation. Kidney Int. 2006; 69:655-62.

21. Pinto M, Dobson S. BK and JC virus: a review. J Infect. 2014;68(Suppl 1):S2-8.

22. Liu S, Chaudhry MR, Berrebi AA, et al. Polyomavirus replication and smoking are independent risk factors for bladder cancer after renal transplantation. Transplantation. 2017;101:1488-94.

23. Papadimitriou JC, Randhawa $\mathrm{P}$, Rinaldo $\mathrm{CH}$, et al. BK polyomavirus infection and renourinary tumorigenesis. Am J Transplant. 2016;16:398-406.

24. Seemayer CA, Seemayer NH, Dürmüller U, et al. BK virus large T and VP-1 expression in infected human renal allografts. Nephrol Dial Transplant. 2008; 23:3752-61.

25. Kenan DJ, Mieczkowski PA, Burger-Calderon R, et al. The oncogenic potential of BK-polyomavirus is linked to viral integration into the human genome. J Pathol. 2015;237:379-89.

26. Monini P, Rotola A, Di Luca D, et al. DNA rearrangements impairing BK virus productive infection in urinary tract tumors. Virology. 1995;214:273-9.

27. Hart A, Smith JM, Skeans MA, et al. OPTN/SRTR 2017 annual data report: kidney. Am J Transplant. 2019;19(Suppl 2):19-123.

28. Hirsch HH, Vincenti F, Friman S, et al. Polyomavirus BK replication in de novo kidney transplant patients receiving tacrolimus or cyclosporine: a prospective, randomized, Treatment with tacrolimus vs. cyclosporine A multicenter study. Am J Transplant. 2013;13:136-45.

29. Binet I, Nickeleit V, Hirsch HH, et al. Polyomavirus disease under new immunosuppressive drugs: a cause of renal graft dysfunction and graft loss. Transplantation. 1999;67:918-22.

30. Manitpisitkul W, Drachenberg C, Ramos E, et al. Maintenance immunosuppressive agents as risk factors for BK virus nephropathy:a casecontrol study. Transplantation. 2009;88:83-8.

31. Bischof $\mathrm{N}$, Hirsch $\mathrm{HH}$, Wehmeier $\mathrm{C}$, et al. Reducing calcineurin inhibitor first for treating BK polyomavirus replication after kidney transplantation: longterm outcomes. Nephrol Dial Transplant. 2019;34:1240-50.

32. Costigliolo F, Lombardo K, Arend $L$, et al. BK virus RNA in renal allograft biopsies. J Histochem Cytochem. 2020; [Epub ahead of print].

33. Kasiske BL, Zeier MG, Chapman JR, et al. KDIGO Clinical Practice Guideline for the care of kidney transplant recipients: a summary. Kidney Int. 2010;77: 299-311.

34. Hirsch HH, Randhawa PS, AST Infectious Diseases Community of Practice. BK polyomavirus in solid organ transplantation-Guidelines from the American Society of Transplantation Infectious Diseases Community of Practice. Clin Transplant. 2019;33:e13528.

35. Yoshimura N, Ushigome $H$, Akioka $K$, et al. The beneficial effect of highdose mizoribine combined with cyclosporine, basiliximab, and corticosteroids on CMV infection in renal transplant recipients. Clin Exp Nephrol. 2013;17:127-33.

36. Ishida H, Takahara S, Amada N, et al. A prospective randomized, comparative trial of high-dose mizoribine versus mycophenolate mofetil in combination with tacrolimus and basiliximab for living donor renal transplant: a multicenter trial. Exp Clin Transplant. 2016; Online ahead of print.
37. Funahashi $Y$, Hattori R, Kinukawa $T$, et al. Conversion from mycophenolate mofetil to mizoribine for patients with positive polyomavirus type BK in urine. Transplant Proc. 2008;40:2268-70.

38. Yuan $X$, Chen $C$, Zheng $Y$, et al. Conversion from mycophenolates to mizoribine is associated with lower BK virus load in kidney transplant recipients: a prospective study. Transplant Proc. 2018;50:3356-60.

\section{Publisher's Note}

Springer Nature remains neutral with regard to jurisdictional claims in published maps and institutional affiliations.
Ready to submit your research? Choose BMC and benefit from:

- fast, convenient online submission

- thorough peer review by experienced researchers in your field

- rapid publication on acceptance

- support for research data, including large and complex data types

- gold Open Access which fosters wider collaboration and increased citations

- maximum visibility for your research: over $100 \mathrm{M}$ website views per year

At BMC, research is always in progress.

Learn more biomedcentral.com/submissions 